\title{
The Transformation of the Environment in the Digital
}

\author{
Age \\ Ekaterina Petrova ${ }^{1, *}$ Ekaterina Gnatik ${ }^{2, a}$
}

\begin{abstract}
${ }^{1}$ Department of Philosophy of Natural Sciences, Institute of Philosophy, Russian Academy of Sciences, Moscow, Russia ${ }^{2}$ Department of Ontology and Epistemology, The Peoples' Friendship University of Russia (RUDN University), Moscow, Russia

aE-mail: ekaterinagnatik@ rambler.ru

*Corresponding author. E-mail: philosophyx@rambler.ru
\end{abstract}

\begin{abstract}
This article analyses the features of the ongoing transformation of the environment impacted by human activities. It is noted that the artificially established digital environment is becoming a habitat of the modern human. In the context of the authors' terminology, the natural environment is a first-order reality, the urbanized environment is a second-order reality, while the digital environment is a thirdorder reality. A third-order reality is ambivalent. On the one hand, it gives the human certain opportunities (educational, cultural, communicational and others), while, on the other hand, it carries a variety of risks (informational stress, cognitive decline etc.). It is the authors' opinion that a gradual change in the intellectual portrait of humankind, which is characterized by emotional intelligence predominance, is becoming the most significant feature of digital environment impact on the human.
\end{abstract}

Keywords: human, information environment, environment, digital age, information society, information and communicational technologies, intelligence, emotional intelligence

\section{INTRODUCTION}

At the current stage, the process of habitat transformation by the human, which has been going throughout the historic times, acquires more and more impressive dimensions, raising serious concerns regarding the unpredictability of its consequences. As far as we know, the habitat of cavemen did not practically differ from the one of other biological species. Our immediate ancestor - Cro-Magnon man lived in a native natural shelter (cave) or a hut made of natural materials, moved on foot, dressed in animal skins and ate benefits of gathering or hunting. In the course of the Homo Sapiens evolution, the natural environment was increasingly pushed out by the artificially established environment: village and then urban settlements, internal environment of dwellings and working places, transport. In order to find oneself in a natural environment, one should "get out in nature": a forest, park, riverbank etc. The modern human lives in an artificially established, urbanized anthropoecosystem rather than natural ecosystem. The

*Fund: The article was prepared as a part or RAS Institute of Philosophy planning research theme "Science, Human Being and Technogenic Civilization Perspectives" № 0156-2019-0002 (Ekaterina V. Petrova) and has been supported by The Russian Foundation for Basic Research (RFBR). Project \# 19-011-00383/19 Bio-and Ecophilosophy in the Modern Culture (Ekaterina N. Gnatik). notion of Umwelt appeared (a term of Jakob Johann von Uexküll), denoting a specific surrounding world, which "any organism adapted to and builds for itself" [1].

If the native, natural environment (a forest, field, river bank and others) could be called a first-order environment, and the urbanized environment artificially established by the human (a city street, flat or office space, car interior) - a second-order environment, in recent years, we have been spending more and more time in a third-order environment. We will attempt to review in more detail what this environment is and how we interact with it.

\section{AMBIVALENCE OF THE THIRD-ORDER} REALITY

The third order environment is an information (digital) environment that was artificially established by the human, namely the Internet, social media, computer games, work with various computer programs starting from typesetting in Microsoft Word and ending with staying in virtual reality, and even trivial watching of TV broadcasts. Certainly, the extent of involvement here differs, and our physical body in this case stays in the second-order reality (or even in the first order reality, as modern technologies allow interacting with the digital reality practically anywhere, including 
nature). It is an entirely different matter with our consciousness that appears to immerse into the thirdorder reality. Moreover, it is immersed so deeply that an individual sometimes stops responding to external stimuli (if, for example, he is heavily involved in a computer game). Accordingly, the artificially established digital environment becomes our habitat and significantly influences us, demanding us to adapt to it.

In recent years, the impact of the information environment has significantly grown. According to the conclusions of psychologists, modern technologies are a core driver in the transformation of higher mental functions in the development conditions of information society. This situation requires the entire scientific community to carry out a thorough analysis and develop various projects of the future [2]. New technologies giving us comfort and unprecedented new opportunities require much in return. This refers to changes in "... the needs structure and motivation", changes in "the way of thinking and lifestyle, skills and even topology of human corporeality" [3].

The opportunities provided by the new digital system are very varied and almost unlimited. These include pure technical solutions related to computerization and production automation, and tremendously grown computing powers, and quick search systems connected to large-scale databases enabling us to get a reply to any question in a matter of seconds. New prospects are also opening up in education: distance learning, access to e-libraries and others. An entire industry of entertainment suitable for every taste that was also generated by the digital reality has emerged. At the same time, so-called Internet surfing contributes to meeting one of the basic instincts, i.e. search for new experiences: "The Internet and computer games become a peculiar surrogate of search activity, replacing it on a subconscious level and, thus, evoking positive emotions [4].

However, there is also a flip "dark" side of the thirdorder environment. This is a negative impact of information flows on our brain, consciousness, worldview and memory. Information could be already detrimental only because it is excessive. When we immerse into the modern digital reality, information overload is practically inevitable. It is no coincidence that such terms as "information pollution", "information noise" and "information stress" have emerged. Information in the form obtained by an average Internet user is often excessive and of poor quality ("information debris"), abounds with unnecessary duplications, so in this information flow it is very difficult to find what we actually need. Information debris of commercial or entertainment nature (advertising, spam, pop-up windows of websites, memes and pictures in social media) distracts, tires out and creates information overload [5]. All these lead to pure medical problems like loss of vision, wrist pains as a result of mouse clicking and keystrokes (this state has even a special term - carpal tunnel syndrome), headaches and neck and back pains due to over-fatigue and long staying in an improper and uncomfortable position.

\section{Change IN The Intellectual PORTRAIT OF HUMANKIND}

However, changes affecting our brains are most worrying. The digital environment most affects the consciousness of the younger generation (children and teenagers), which has not fully formed and, therefore, is especially vulnerable. It is ironic that children and adolescents immerse into the third order reality especially deep. As A.V. Markov stressed, "... the human brain (particularly, the child's brain) is specifically adapted to mastering, executing and subsequent transferring of instructions to other people that are "recorded" with the help of the means of communication inherent to the human" [6]. It is known that in child development there are special periods, some kind of "open windows", when a child is most susceptible to the perception of outside world's influence and must develop certain skills (speech, skills of social communication with other individuals). However, if during these periods, he mainly interacts with modern means of mass communication and information (television, tablet, and smart phone) rather than his parents, other adults and children, the consequences could be catastrophic. The example described by outstanding culturologist Vyacheslav Vsevlodovich Ivanov in his work Odd and Even. The Asymmetry of Brain and Sign Systems is very illustrative here. It is noteworthy that this work was published in 1968, i.e. long before the modern "information explosion". However, the disturbing trends were traced even at that time. This refers to a little child who was not yet able to speak, whose parents going to work left him alone in front of a TV set on for a long time. As a result, the child appeared to show up some features that indicated to a deviation in his development: "he did not say anything, but began writing and, in particular, he could write the names of TV programs and texts of commercial advertisements, ... when the boy began speaking, he pronounced the names of individual car makes rather than whole sentences " [7].

Vyacheslav Ivanov associates this disorder with a huge volume of spatial-visual information falling on the child from the TV screen every and each day, which has slowed the left hemisphere development and, therefore, language development. It is also possible that something similar to the "Mowgli syndrome" has shown up in this situation. It is known that there is a 
critical age in the development of child's psyche, namely up to 5 years (and according to other data, up to 6 years). If at that very time, a child is precluded from interacting with his parents or other people (known cases of "Mowgli children", who were brought up by animals), on a later stage he will not master speech and other skills of human communication. In that particular case, a surrogate replaced a full verbal contact with the parents, namely TV viewing, which, of course, is not so critical as upbringing by animals, but could not be a full substitute.

In his book The Anti-Brain: Digital Technologies and Brain, Manfred Spitzer, a German psychiatrist, also underlines a fatal role of abuse of interaction with the third order environment. Based on his research, he made a conclusion that the more children watch TV, the more their language development slows, and even worse would be their education in adulthood. Even special children's educational movies and programs do not facilitate learning, but prevent it. The researcher claims that uncontrolled immersion into the information reality of the third order may lead to so-called "digital dementia". It is possible to draw an analogy between our brain and muscles: if one actively exercises the brain, it grows (a number of neural connections increase, and even its separate areas, like hippocampus, may increase). An effective brain training includes reading of long complex texts, orienteering in a new area, deep immersion into a topic and development of critical thinking skills. The more superficially a person perceives information, the fewer synapses are activated, and the worse the person adsorbs this information. Socalled "mosaic thinking" emerges: it is difficult for a human to focus on the perception and analysis of long texts, solution of logical problems, he "nibbles at the edges", perceives scattered and patchy bits and pieces of information that change each other like pictures in a kaleidoscope. This affects learning abilities, holistic worldview formation and development of independent critical thinking.

Could it be so that the modern information environment is good because it provides wide opportunities for socialization? Indeed, without the information technology interference, a circle of communication of a modern human is limited to several friends and relatives, a dozen other acquaintances, neighbors and work-fellows. In social media, the number of "friends" could reach several thousands. However, we agree with M. Spitzer: "Social media do not contribute in any way either to the expansion or deepening of contacts. Their only result is social isolation and superficial contacts" [8].

It would seem that the presence of huge databases, information storages and a possibility to quickly search for information is an undoubted advantage of the modern digital environment. There is no need to overload any more your memory with a wealth of information that is not known when it would be useful, or might not be useful at all. However, here, too, the situation is ambiguous. Researchers note worrisome changes in our natural intelligence that occur under the impact of information and communication technologies: "The "externalization" of memory takes place, human abilities to remember and logical abilities weakens, the formation of "mosaic consciousness" becomes an obstacle to abstract thinking development, and the ease of access to information supersedes the need for individual knowledge production" [9].

The matter is that our memory is not a "dump" for scattered information gigabytes. Various information about the surrounding world, which we come to know in the process of individual development, are structured, connected with each other and ultimately form the basis for holistic worldview development. If we do not keep in mind a certain amount of knowledge hoping that we may "Google" it at any time, any holistic worldview formation is out of question.

Psychologists identify the state of a modern human, when conventional information and communication means are refused or inaccessible, as invalidization. [10]. In other words, for a modern human this state is so painful and difficult to tolerate that it could be compared with a restriction or loss of the functions of his organs or body parts.

Despite the ambivalence of the impact on the digital environment human, one thing is certain: the use of computer technologies significantly transforms the mental, motivational and emotional components of the human person [11]. Most likely, many cognitive functions like the ability to store vast amounts of information, planning, modeling and "computational" intelligence will be increasingly "farmed out" to technologies. Some other, pure human abilities, which, at least for the time-being, could not be replaced by machines, will come to the fore. This refers to so-called emotional intelligence (EI). P. Salovey and J. Mayer were the first to introduce the "emotional intelligence" concept in 1990, and they defined it as a group of mental abilities that provide awareness and understanding of own emotions and emotions of surrounding people. Highly developed emotional intelligence provides advantages in professional activities and interpersonal relations, facilitates selfknowledge and, ultimately, greater awareness and mental health: «"individuals with a high level of emotional intelligence development possess evident abilities to understand their own emotions and emotions of other people, as well as abilities to manage the emotional sphere, which specify greater adaptability and effectiveness in communication" [12]. 


\section{CONCLUSION}

Information and communication technologies invade our lives and change them so quickly that we are not able to adapt and respond to these developments. A decade earlier, the scale and depth of the impact by the third order reality on the human were not so obvious to all scientists and philosophers. The questions were raised: "Why is the adaptation to the artificial information environment needed at all?" Over the past years, in our opinion, the relevance of the problem became obvious to everyone: "The electronic culture changes the human who, in turn, evolves in a way to change the culture" [13]. A series of publications that analyze the problem of human transformation and adaptation in the digital age from the perspective of medicine, sociology, economics, cultural studies and others have been issued. At the same time, these raises some other issues like fragmentation, multidimensionality of these works, and lack of a holistic understanding of the problem. It seems to us that philosophy must play its role here as the most general reflection. The human problem is always in the center, and it is the philosophy that possesses all the opportunities for a comprehensive analysis of this problem. However, one should avoid any extremes like allowing of uncontrolled immersion into the third order reality, on the one hand, and a rigorous restrictive policy, on the other hand. Freedom of action in the digital environment must be perceived as a felt-need rather than permissiveness. The opportunities of the new digital world are unquestionable; however, they should not facilitate dehumanization by way of substituting basic human values. "Mosaic thinking" should not oust a free, systemic mental process. As an example, for this purpose in the education sector, it is required to structure a modern learning process properly starting from early development classes for preschoolers and ending with higher school. Both conventional and modern information and communication technologies must be combined properly in education.

In our view, a gradual change in the intellectual portrait of humankind is becoming the most significant feature of digital environment impact on the human, so an increasingly important role in this portrait will be played by emotional intelligence (EI) rather than intelligence (mental abilities), which will be gradually ousted by superior artificial intelligence.

\section{References}

[1] E.N. Knyazeva, The Concept of "Umwelt" of Jakob Johann von Uexküll and its Significance for Modern Epistemology. The Problems of Philosophy. 2015. \#. 5. p. 30.

[2] V.M. Rosin, The Discourses and Types of the Future. Epistemology and Philosophy of Science." 2017. V. 52. \#. 2. p. 149.
[3] V.A. Yemelin, E. I. Rasskazova, A.Sh. Tkhostov, The Psychological Consequences of Information Technology Development. The National Psychological Journal. 2012. \#. 1 (7). p. 81.

[4] S.O. Gapanovich, V.F. Levchenko, On the Problem of Information Anthropoecology. The Principles of Ecology. 2017. \#. 4. p. 7.

[5] L.V. Bayeva, The Electronic Culture: Experience of Philosophical Analysis. The Problems of Philosophy. 2013. \#. 5. pp. 75-83.

[6] A.V. Markov, The Human Evolution. In 2 books. Book 2. Monkeys, Neurones and Soul. Moscow: AST: Corpus, 2011. 511 pages. p. 360 .

[7] Vyach. Vs. Ivanov, Odd and Even. The Asymmetry of Brain and Sign Systems. Moscow: The Soviet Radio, 1978. 184 pages. p. 51 .

[8] Manfred Spitzer, The Anti-Brain: Digital Technologies and Brain, Moscow:. AST, 2014. 288 pages. p. 23

[9] I.Yu. Alekseyeva, E.A. Nikitina, Intelligence and Technology. Moscow: Prospekt, 2015. 96 pages. p. 5.

[10] V.A. Yemelin, E.I. Rasskazova, A.Sh. Tkhostov, p. 82.

[11] Ekaterina Gnatik, Information Technologies in Educational Sphere: Challenges and Risks. Advances in Social Science, Education and Humanities Research, Volume 232. 4th International Conference on Arts, Design and Contemporary Education (ICADCE 2018). Paris: Atlantis Press, 2018. pp. 584 587.

[12] I.N. Andreyeva, Emotional Intelligence: Phenomenon Studies. The Problems of Psychology. 2006. \#. 3. pp. 78-86.

[13] N.A. Kasavina, The Human and Engineering: Ambivalence of Electronic Culture. The Epistemology and Philosophy of Science." 2018. V. 55. \#. 4. p. 138. 